\title{
What is the evidence base for fluid resuscitation in acute medicine? 滥
}

\author{
Authors: Adam Seccombe ${ }^{A, *}$ and Elizabeth Sapey ${ }^{B, *}$
}

Intravenous fluids are commonly prescribed but uncertainty remains about how to assess when fluids are required and how much to give, particularly in our multimorbid, polymedicated and ageing population. Furthermore, studies have noted that fluid resuscitation can be harmful even if clinical evidence of hypervolaemia is not present. Two recent guidelines have acknowledged a limited evidence base to guide fluid assessment. A recommended means to assess hypovolaemia includes assessment of fluid responsiveness. Fluid responsiveness is a rise in stroke volume following an increase in preload, achieved using a fluid challenge or a passive leg raise. However, the means of defining fluid responsiveness and its ability to identify patients who would benefit from fluid resuscitation is currently unclear. This review discusses the current guidelines about, and the evidence base for the provision of, intravenous fluids in the acutely unwell medical patient. It highlights how little evidence is available to guide medical practice.

KEYWORDS: Fluid resuscitation, fluid assessment, fluid responsiveness, passive leg raise, water-electrolyte balance, fluid therapy

\section{Introduction}

'Medicine is a science of uncertainty and an art of probability.' Sir William Osler (1849-1919).

Intravenous (IV) fluid is one of the most commonly prescribed hospital treatments. Despite its low cost ${ }^{1}$ the annual NHS spend on IV fluid is in excess of $£ 156$ million. ${ }^{2}$ IV fluid use in some resuscitation scenarios, such as traumatic blood loss, is well evidenced. ${ }^{3}$ In acute medical scenarios such as shock, the benefits are less well documented, although fluid therapy is highly recommended in many guidelines and reviews, for example in a recent review which stated that 'fluid therapy... is an essential part of the treatment of any form of shock'. ${ }^{4}$ Acute kidney injury guidelines advise that we should identify and correct hypovolaemia through 'adequate' fluid replacement, ${ }^{5}$ and international sepsis guidelines state that the use of IV fluid 'is a cornerstone of modern

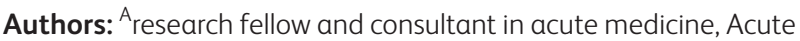
Medicinal Unit, University Hospital Birmingham NHS Foundation Trust, Birmingham, UK; ${ }^{B}$ reader and consultant in respiratory and general medicine, Institute of Inflammation and Ageing, University of Birmingham, Birmingham, UK; *both authors contributed equally therapy'. ${ }^{6}$ Despite this, evidence to describe the indications, dose and rate of administration of IV fluid is lacking, as is the use of IV fluid over the course of a patient's illness. Medical practitioners currently rely on their clinical acumen alone to guide prescribing practices, akin to the 'science of uncertainty and art of probability' described by Sir William Osler in the last century.

Common practice is to use the clinical features of hypovolaemia and hypervolaemia to signal when treatment should be started and stopped. Such features are well described; ${ }^{7}$ however, none are specific to volume status ${ }^{8}$ and many are not easy to assess. ${ }^{9}$ A recent systematic review of 30 studies found that clinical features (including hypotension and tachycardia) were not reliable predictors of hypovolaemia. ${ }^{10}$ The same is true of features of hypervolaemia, which are present in many conditions. ${ }^{8}$ Even if it could be diagnosed accurately, hypervolaemia due to excess fluid is an iatrogenic overdose and should not be used as a marker to stop fluid administration. In support of this, a recent trial in Zambia randomised hypotensive, septic adults to a usual care group (IV fluid determined by the treating clinician) or a sepsis protocol (aggressive IV fluid limited only by clinical signs of hypervolaemia, alongside vasopressors and blood transfusion when indicated). ${ }^{11}$ Use of the sepsis protocol led to a significant increase in in-hospital mortality (48.1\%) compared to usual care (33.0\%). However, a high proportion of participants had human immunodeficiency virus (89.5\%) making it unclear whether these results can be extrapolated to a general medical population.

Of concern, harm from fluids has been demonstrated even in the absence of hypervolaemia. The FEAST (Fluid Expansion As Supportive Therapy) trial, a randomised controlled trial involving East African children with a severe febrile illness, noted that fluid boluses (using either $0.9 \%$ saline or albumin) were associated with an excess three deaths in every hundred patients, compared to conservative maintenance fluids alone. ${ }^{12}$ A reanalysis of this study suggested that cardiovascular collapse was linked to the increased mortality rather than respiratory failure due to pulmonary oedema. ${ }^{13}$ Despite the lack of high-quality IV fluid trials in developed world populations, there is a signal of harm including complications noted in one in five patients in the NHS. ${ }^{14}$ Consistent with this, observational studies performed in an intensive care setting have found associations between a positive fluid balance and increased mortality in inflammatory conditions and AKI. ${ }^{15-18}$

\section{Patient variability and fluid assessment}

Variations in patient physiology and pathology impact upon the clinical assessment of fluid status, as summarised in Fig 1. 
Fig 1. Factors that can affect a fluid assessment. Four groups of factors are depicted that affect a patient's fluid balance and therefore a fluid assessment. These factors are further broken down into subgroups which illustrate the potential complexities involved.

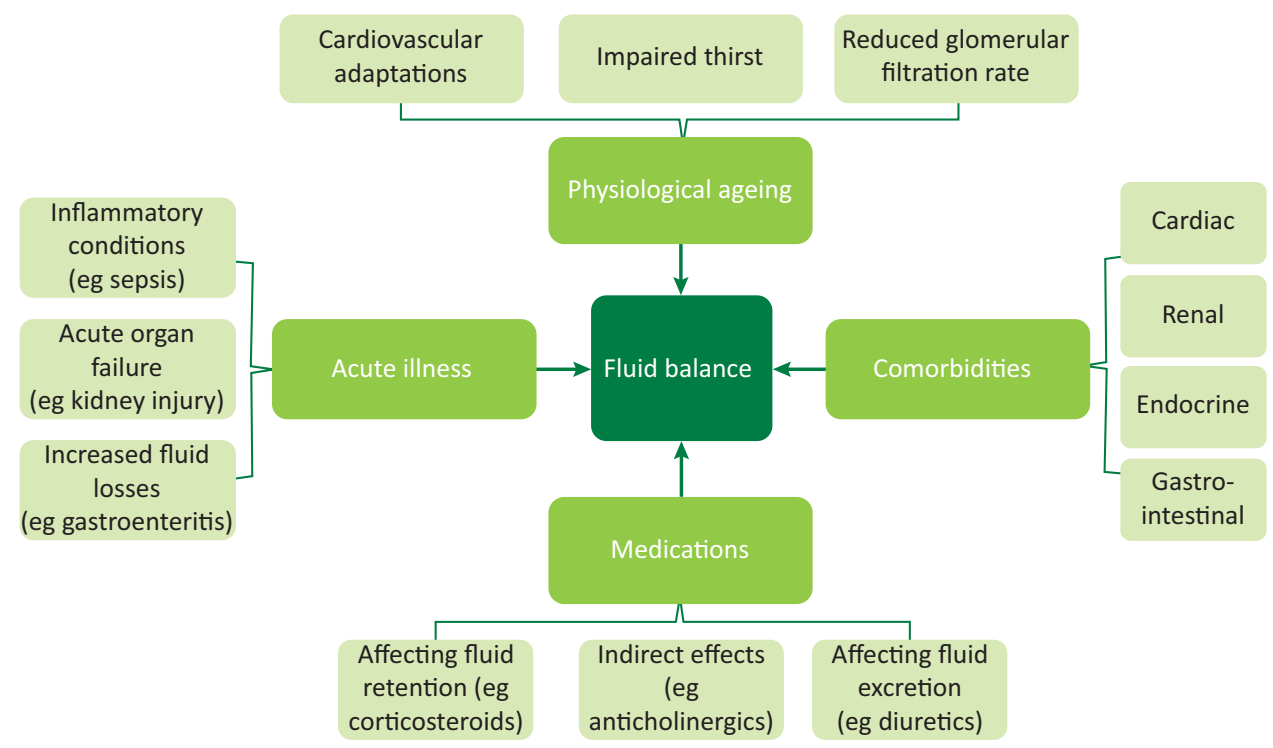

Physiological ageing impacts organ systems and the 2015 Cochrane review noted there was 'limited evidence of the diagnostic utility of any individual clinical symptom, sign or test, or combination of tests, to indicate water-loss dehydration in older people. ${ }^{, 19}$ For example, maximal cardiac output declines with age, even in the absence of hypertension or cardiovascular disease. ${ }^{20} \mathrm{~A}$ decrease in heart rate response is seen to stressors, which correlates with a reduced responsiveness of $\beta$-adrenoceptors to adrenergic stimuli, blunting the tachycardia that can identify hypovolaemia. This decline is partially compensated by an increase in the venous return, as the $\alpha$-adrenoceptor-mediated venoconstriction is less affected by ageing. ${ }^{21}$

This physiological variability is further accentuated by comorbidities. Chronic organ dysfunction alters fluid homeostasis. For example, a reduced cardiac output in heart failure causes inappropriate activation of the renin-angiotensin system and a consequent expansion of the extracellular fluid compartment. ${ }^{22}$

Medications used to manage chronic disease can also alter fluid balance, by direct action, eg side effects such as diarrhoea and vomiting, or through indirect means. Anticholinergic medications block parasympathetic signals to the salivary glands, causing xerostomia. If mild, this can lead to an increased fluid intake to moisten the mouth. Conversely, if severe, xerostomia can interfere with a patient's ability to swallow and prevent adequate hydration. ${ }^{23}$

Fluid assessment is made yet more complex in the presence of acute illness and injury. ${ }^{6}$ For example, sepsis affects the cardiovascular system in multiple ways. Hypovolaemia occurs due to direct fluid loss, including insensible losses due to fever. Sepsis has a direct inhibitory impact on cardiac function, reducing contractility and limiting cardiac output. ${ }^{24}$ Vasodilatation causes a reduction in the systemic vascular resistance and, if severe, a fall in blood pressure. Sepsis has also been shown to damage the endothelial glycocalyx, a network of proteoglycans and glycoproteins covering the luminal vascular endothelium allowing fluid leak into the extracellular compartment. ${ }^{25}$

All too commonly, patients attending hospital for unplanned, acute medical care have a combination of these factors. They are old, multimorbid, polymedicated and have an acute deterioration in status, making the assessment of fluid status exceptionally challenging.

\section{Recommendations from guidelines}

In the last decade, two major guidelines have attempted to address the uncertainty regarding fluid assessment.

The Surviving Sepsis Campaign (SSC) took a prescriptive approach to fluid use in sepsis, advising a fixed dose once septic shock is identified. ${ }^{6}$ It 'strongly' recommends $30 \mathrm{~mL} / \mathrm{kg}$ of IV fluid within 3 hours in patients who meet the criteria for septic shock (hypotension or lactate $\geq 4 \mathrm{mmol} / \mathrm{L}$ ). Despite this advice, the guideline acknowledges the evidence for their recommendation is weak, noting 'there is little available evidence from RCTs to support its [IV fluid] practice'.

The recommendation is based on the results of a single-centre, unblinded trial involving 263 septic patients. ${ }^{26}$ It found a $16 \%$ mortality reduction when an 'early goal-directed therapy' (EGDT) protocol was used in place of usual care. However, three subsequent trials, involving a combined total of 4,175 patients, found no benefit of EGDT compared to usual care. ${ }^{27-29}$ Furthermore, a retrospective cohort study found that $67 \%$ of patients had evidence of fluid overload at 24 hours when EGDT recommendations were followed, with a corresponding $92 \%$ increased risk of mortality. ${ }^{30}$

If initial fluid resuscitation is followed by ongoing hypotension or hyperlactataemia, the SSC guideline recommends the use of physiological variables to determine the need for additional IV fluid. ${ }^{6}$ Recommended variables include central venous pressure (CVP), central venous oxygen saturation $\left(\mathrm{S}_{\mathrm{CV}} \mathrm{O}_{2}\right)$, bedside echocardiography and a dynamic assessment of fluid responsiveness. However, these variables measure different physiological processes. A rise in CVP can be a marker of fluid excess in the venous compartment. $\mathrm{S}_{\mathrm{Cv}} \mathrm{O}_{2}$ is a surrogate for the balance between oxygen delivery and consumption. Echocardiography allows the measurement of cardiac contractility and can estimate venous pressures. Fluid responsiveness, discussed in more detail below, describes an increase in cardiac performance 
Table 1. NICE recommended parameters to indicate hypovolaemia or shock ${ }^{a}$

\begin{tabular}{|c|c|c|}
\hline Parameter & $\begin{array}{l}\text { Indicator of } \\
\text { hypovolaemia }\end{array}$ & Indicator of shock \\
\hline $\begin{array}{l}\text { Systolic blood } \\
\text { pressure }\end{array}$ & $<100 \mathrm{mmHg}$ & $\begin{array}{l}<20 \mathrm{mmHg} \text { below } \\
\text { baseline }\end{array}$ \\
\hline Heart rate & $>90$ beats $/ \mathrm{min}$ & $\begin{array}{l}\text { >20 bpm above } \\
\text { baseline }\end{array}$ \\
\hline Capillary refill time & $>2 \mathrm{~s}$ & $>2 \mathrm{~s}$ \\
\hline Respiratory rate & $>20$ breaths/min & $>20$ breaths/min \\
\hline $\begin{array}{l}\text { National Early } \\
\text { Warning Score }\end{array}$ & $>4$ & Not mentioned \\
\hline Urine output & Not mentioned & $<0.3 \mathrm{~mL} / \mathrm{kg} / \mathrm{h}$ \\
\hline Passive leg raise & $\begin{array}{l}\text { Fluid responsiveness } \\
\text { demonstrated }\end{array}$ & Not mentioned \\
\hline
\end{tabular}

${ }^{a}$ This table summarises two sets of criteria that are listed at different points in NICE Clinical Guideline 174. ${ }^{4}$ One set of criteria is suggested for the diagnosis of hypovolaemia and the other as a means of diagnosing any form of shock.

$\mathrm{h}=$ hour; NICE = National Institute for Health and Care Excellence; $s=$ seconds

following a fluid bolus. There is no recommendation on which assessment tool to use or whether combining these variables may help determine fluid status. Furthermore, many of these variables would be challenging to measure in an acute medical setting.

The latest National Institute for Health and Care Excellence (NICE) guideline for the recognition, assessment and early management of sepsis adopted a more conservative use of IV fluid in sepsis. ${ }^{31}$ It advocated up to $1000 \mathrm{~mL}$ of IV fluid, if indicated, before senior involvement. To determine whether IV fluid was indicated, it recommended the use of the 2013 NICE guideline for IV fluid use in adults, which included guidance to identify hypovolaemia defined simply as a reduced circulating volume. ${ }^{14}$ The diagnostic ability of the parameters within this guidance (as described in Table 1) is questionable. The National Early Warning Score (NEWS) is the recommended early warning score in the UK to identify acutely unwell patients. It, along with tachypnoea, is a marker of acuity, not hypovolaemia. There is little data to support the use of capillary refill time (CRT) in adults and the normal value of fewer than 2 seconds is an arbitrary figure. ${ }^{32}$ One study described the $95 \%$ confidence limit for a normal CRT in adults aged over 62 years extends up to 4.5 seconds, raising questions about the recommended cut-off. ${ }^{33}$

The guideline subsequently described a different set of parameters to identify shock, defined as a 'life-threatening condition with generalized maldistribution of blood flow causing failure to deliver and/or utilize adequate amounts of oxygen, leading to tissue dysoxia.. ${ }^{14}$ If present, the guideline suggests urgent fluid resuscitation to restore vascular volume. While shock can be secondary to hypovolaemia, it can also be caused by conditions where fluid may be harmful, eg cardiac failure.

\section{Fluid responsiveness}

A test for hypovolaemia that is mentioned in both the SSC and NICE guidelines is fluid responsiveness. If a physiological variable

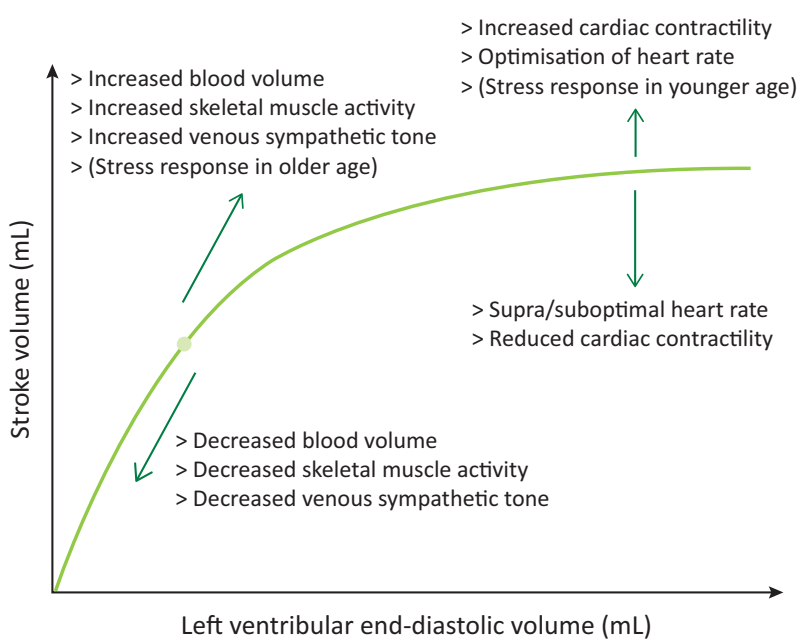

Fig 2. The Frank-Starling Curve. This schematic of the Frank-Starling curve describes the effects of an increasing preload on the stroke volume and the factors that can alter this relationship. Some of these factors change a person's position on the curve by altering the preload (diagonal arrows) and others change the shape of the curve by altering the cardiac function directly (vertical arrows).

(eg stroke volume) increases following an increase in preload, then the patient is defined as fluid responsive. The concept is based on the Frank-Starling curve (Fig 2), ${ }^{34}$ which documents the relationship between stroke volume and left ventricular end-diastolic volume (LVEDV) - a measure of preload. Multiple, circulating volume-independent factors can alter the LVEDV including age, multimorbidity, medications and acute illness. ${ }^{35}$ Furthermore, fluid responsiveness is seen in healthy volunteers. ${ }^{36,37}$ When 3 litres of IV fluid were administered to healthy adults over 3 hours, the cardiac index increased by as much as 30\% suggesting it is a normal physiological condition to be a fluid responder. ${ }^{37} \mathrm{No}$ studies have differentiated fluid responsiveness due to normal physiology from that seen in hypovolaemia.

Evidence to support the use of fluid responsiveness in the acutely unwell patient is mixed. A review suggested that $50 \%$ of patients who are given fluid resuscitation in an intensive care setting are not fluid responsive. ${ }^{38}$ The use of fluid responsiveness to guide IV fluid administration was linked to reduced mortality in a systematic review of 13 trials in a postsurgical, intensive care population. ${ }^{39}$ However, a different systematic review of 23 trials using fluid responsiveness to guide perioperative IV fluid use found no difference in mortality with usual care, although it noted a reduction in postoperative complications. ${ }^{40} \mathrm{~A}$ smaller systematic review based in the emergency department included two randomised controlled trials which noted no difference in surrogate outcomes (lactate clearance and SOFA score) between a fluid responsiveness protocol and usual care. ${ }^{41}$ Furthermore, studies have noted the response to a fluid bolus is transient. One study noted the cardiac output returned to baseline values after 90 minutes in fluid responsive patients, the majority of whom had septic shock. ${ }^{42}$

A fluid challenge is the most cited approach to increase preload when assessing for fluid responsiveness. No consensus exists regarding the type, rate and amount of fluid that should be used. A recent systematic review of 71 studies explored how a 


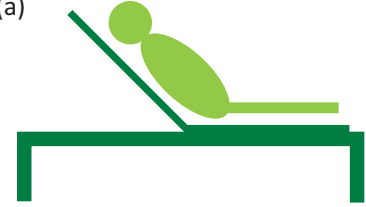

(b)

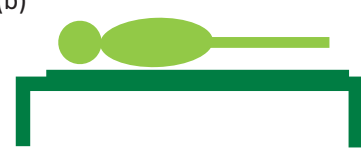

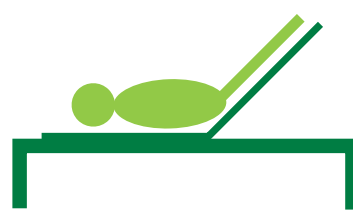

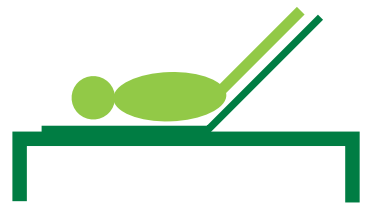

Fig 3. Two methods of a passive leg raise. There are two techniques described for a passive leg raise (PLR) in the literature: (a) a semi-recumbent PLR starts at $45^{\circ}$ and the patient is tilted backwards until the torso is horizontal leaving the legs are at $45^{\circ}$; (b) a supine PLR starts with the patient in a supine position and the legs are elevated to $45^{\circ}$

fluid challenge has been defined with $75 \%$ of studies using 500 $\mathrm{mL}$ of fluid and $62 \%$ using colloids. ${ }^{43}$ More recently, 'mini-fluid challenges' of $100 \mathrm{~mL}$ have been shown to predict the effects of larger fluid challenges. ${ }^{44}$ The rate of fluid also varied widely between 5 and 90 minutes per bolus, with $45 \%$ of studies giving fluid over 30 minutes. This wide heterogeneity is a barrier to meaningful comparisons between studies.

Apart from a fluid challenge, two techniques to increase preload and identify the presence or absence of fluid responsiveness have been studied: pulse pressure variation (PPV) and the passive leg raise (PLR). The size of variations in pulse pressure with respiration can be an accurate marker of response to a fluid challenge in stable, perioperative patients. ${ }^{45}$ However, these measurements are poor predictors of fluid responsiveness in less controlled scenarios, such as intensive care. ${ }^{46} \mathrm{PPV}$ is therefore unlikely to be of use in acute medicine.

A PLR describes any manoeuvre in which the legs are elevated. This can be achieved by tilting the bed from a semi-recumbent position (Fig 3a) or elevating the legs from a supine position (Fig 3b). These manoeuvres lead to a transfer of blood from the legs and abdomen into the thorax and a resultant increase in preload. Radionuclide imaging estimates that the volume of blood in the calves of healthy volunteers, before and after the legs were raised to $20^{\circ}$ was reduced by $150 \mathrm{~mL} .^{47}$ This volume will be increased once the movement of blood from the thigh and abdomen is taken into account. The increase in preload from a PLR is completely reversed once the legs are lowered making this a safe manoeuvre for patients at risk of fluid overload. ${ }^{48}$

Systematic reviews have looked at the ability of a PLR to mimic the effects of a fluid bolus. ${ }^{49}$ Despite studies using different volumes and forms of fluid challenge and different methods to assess cardiac output, there was a mean PLR-associated change in cardiac output in those subjects who also increased their cardiac output following a fluid challenge, with no difference between patients who were spontaneously breathing and those on a ventilator. ${ }^{49}$ These results suggest that PLR could be used in acute medical patients as an assessment of fluid responsiveness but it remains unclear what action this test should prompt. It is unknown what proportion of acutely unwell adults will have evidence of fluid responsiveness as measured by $P L R$, whether giving fluids to those

with a positive PLR will improve outcomes, whether PLR can be used to determine when to stop fluid resuscitation or indeed if PLR is the best (and most pragmatic) means to assess fluid status. As with a fluid challenge, there are a wide variety of studied methods for measuring fluid responsiveness. This heterogeneity has been recognised with one review suggesting the following definition.

An increase in a physiologic parameter, preferably cardiac output, within 15 minutes superseding twice the error of the measuring technique after a 15-minute administration of $6 \mathrm{~mL}$ / $\mathrm{kg}$ of crystalloids ${ }^{50}$

This reflects the uncertainty regarding how fluid responsiveness should be assessed.

\section{Avenues for future research}

The evidence base for most medical conditions is expanding, including powerful insights into the potentially negative consequences of medical practices which were advocated in the past. IV fluids are commonly given to acutely unwell adults based on the probability of benefit despite evidence of harm in some studies. We still do not know when to give fluids (or when to withhold them), how much to give or how to accurately assess the response, especially in our ageing, multimorbid and polymedicated patients. Building an evidence base to help clinicians use IV fluid appropriately is essential.

While there is awareness of the complications of IV fluid therapy, there is a limited understanding of the incidence of these complications and their impact on outcomes. The need to improve our understanding of the harms related to IV fluid has been acknowledged by the NICE guideline and forms a key research recommendation. ${ }^{14}$

How the medical profession currently performs a fluid assessment is ill-defined. Our inability to describe current practice prevents trials from differentiating between effective and ineffective assessment techniques when usual care is a treatment option. Qualitative analysis to explore the reasons that clinicians give fluid resuscitation would clarify practice and may improve protocol breaches that are common when fluid resuscitation protocols are studied. ${ }^{51}$

Studies have not compared patient outcomes when fluid responsiveness is compared to other means to assess fluid status, such as CVP. Current evidence has shown that assessment tools such as CVP are poor predictors of fluid responsiveness, but not that they are less effective than fluid responsiveness at predicting whether IV fluid is beneficial. The evidence base supporting fluid responsiveness is limited by heterogeneity in definition and practice. It will remain challenging to study hypovolaemia if potential assessment tools have no agreed definition.

\section{Summary}

The provision of IV fluid is one of the most common therapeutic interventions, but there is the potential for harm as well as benefit and the ongoing expansion of our ageing, multimorbid and polymedicated population will only make fluid assessment more challenging. The evidence to support when and how to prescribe fluids is limited. Therefore; robust, evidence-based recommendations for the use of fluid resuscitation by the acute physician are not currently possible. Instead, there are a high 
number of review articles and educational pieces which rely upon expert opinion and usual practice. Such paucity of data is hard to justify in our era of evidence-based practice and there is a clear need for more research to guide how fluid resuscitation should be used in the acutely unwell patient.

Returning to the quote by Osler, given the heterogeneity of medical patients, we may not be able to turn uncertainty into certainty, but the probability of benefit from IV fluids should be based more on science and less on art.

\section{Author contribution}

Dr Seccombe and Dr Sapey jointly wrote the article and Dr Sapey finalised the article for publication.

\section{Conflicts of interest}

Dr Sapey reports grants from Alpha 1 Foundation, grants from Glaxo Smith Kline, grants from Medical Research Council, grants from NIHR, grants from Wellcome Trust and grants from British Lung Foundation, outside the submitted work. Dr Seccombe has nothing to declare.

\section{References}

1 Joint Formulary Committee. British National Formulary. 74th edn, London: BMJ Group and Pharmaceutical Press, 2017.

2 National Institute for Health and Care Excellence. Intravenous fluid therapy in adults in hospital: Costing Statement. CG174. London: NICE, 2013.

3 Mapstone J, Roberts I, Evans P. Fluid resuscitation strategies: a systematic review of animal trials. J Trauma 2003;55:571-89.

4 Vincent JL, De Backer D. Circulatory shock. N Engl J Med 2013;369:1726-34.

5 Kidney Disease Improving Global Outcomes. Clinical Practice Guideline for Acute Kidney Injury. Kidney Int Supplements 2012;2:1-138.

6 Rhodes A, Evans LE, Alhazzani W et al. Surviving Sepsis Campaign: International guidelines for management of sepsis and septic shock: 2016. Intensive Care Med 2017:43:304-77.

7 Frost $P$. Intravenous fluid therapy in adult inpatients. BMJ 2015;350:g7620.

8 McGee S. Evidence-Based Physical Diagnosis, 4th edn. Philadelphia, PA: Elsevier, 2018.

9 Cook DJ. Clinical assessment of central venous pressure in the critically ill. Am J Med Sci 1990;299:175-8.

10 Pacagnella RC, Souza JP, Durocher ] et al. A systematic review of the relationship between blood loss and clinical signs. PLoS One 2013;8:e57594.

11 Andrews B, Semler MW, Muchemwa L et al. Effect of an early resuscitation protocol on in-hospital mortality among adults with sepsis and hypotension: a randomized clinical trial. JAMA 2017;318:1233-40.

12 Maitland K, Kiguli S, Opoka RO et al. Mortality after fluid bolus in African children with severe infection. N Engl J Med 2011;364:2483-95.

13 Maitland K, George EC, Evans JA et al. Exploring mechanisms of excess mortality with early fluid resuscitation: insights from the FEAST trial. BMC Med 2013;11:68

14 National Institute for Health and Care Excellence. Intravenous fluid therapy in adults in hospital. CG174. London: NICE, 2013.

15 Vincent JL, Sakr Y, Sprung CL et al. Sepsis in European intensive care units: results of the SOAP study. Crit Care Med 2006;34:34453.

16 Sadaka F, Juarez M, Naydenov S, O'Brien J. Fluid resuscitation in septic shock: the effect of increasing fluid balance on mortality. J Intensive Care Med 2014;29:213-7.
17 The National Heart, Lung and Blood Institute Acute Respiratory Distress Syndrome Clinical Trials Network et al. Comparison of two fluid-management strategies in acute lung injury. N Engl $]$ Med 2006;354:2564-75.

18 Brotfain E, Koyfman L, Toledano R et al. Positive fluid balance as a major predictor of clinical outcome of patients with sepsis/septic shock after ICU discharge. Am J Emerg Med 2016;34:2122-6.

19 Hooper L, Abdelhamid A, Attreed NJ et al. Clinical symptoms, signs and tests for identification of impending and current waterloss dehydration in older people. Cochrane Database Syst Rev 2015:CD009647.

20 Houghton D, Jones TW, Cassidy S et al. The effect of age on the relationship between cardiac and vascular function. Mech Ageing Dev 2016;153:1-6.

21 Tonner PH, Kampen J, Scholz J. Pathophysiological changes in the elderly. Best Pract Res Clin Anaesthesiol 2003;17:163-77.

22 Guyton AC, Hall JE. Textbook of medical physiology, 10th edn. Philadelphia, PA: WB Saunders, 2000.

23 Gilbert GH, Heft MW, Duncan RP. Mouth dryness as reported by older Floridians. Community Dent Oral Epidemiol 1993;21:390-7.

24 Remick DG. Pathophysiology of sepsis. Am J Pathol 2007;170:143544.

25 Clough G. Relationship between microvascular permeability and ultrastructure. Prog Biophys Mol Biol 1991;55:47-69.

26 Rivers E, Nguyen B, Havstad S et al. Early goal-directed therapy in the treatment of severe sepsis and septic shock. N Engl J Med 2001;345:1368-77.

27 ProCESS-Investigators. A randomized trial of protocol-based care for early septic shock. N Engl J Med 2014;370:1683-93.

28 Mouncey PR, Osborn TM, Power GS et al. Trial of early, goal-directed resuscitation for septic shock. N Engl J Med 2015;372:1301-11.

29 Arise Investigators. Goal-directed resuscitation for patients with early septic shock. N Engl J Med 2014;371:1496-506.

30 Kelm DJ, Perrin JT, Cartin-Ceba R et al. Fluid overload in patients with severe sepsis and septic shock treated with early goaldirected therapy is associated with increased acute need for fluid-related medical interventions and hospital death. Shock 2015;43:68-73.

31 National Institute for Health and Care Excellence. Sepsis: recognition, assessment and early management. NG51. London: NICE, 2016.

32 Lewin J, Maconochie I. Capillary refill time in adults. Emerg Med J 2008;25:325-6.

33 Schriger DL, Baraff LJ. Capillary refill - is it a useful predictor of hypovolemic states? Ann Emerg Med 1991;20:601-5.

34 Starling $E H$, Visscher MB. The regulation of the energy output of the heart. J Physio 1927;62:243-61.

35 Levy MN. Peripheral circulation and its control. In: Berne RM, Levy MN, Koeppen BM, Stanton BA (eds), Physiology. St Louis, USA: Mosby, 2004:380-94.

36 Godfrey GE, Dubrey SW, Handy JM. A prospective observational study of stroke volume responsiveness to a passive leg raise manoeuvre in healthy non-starved volunteers as assessed by transthoracic echocardiography. Anaesthesia 2014;69:306-13.

37 Kumar A, Anel R, Bunnell E et al. Pulmonary artery occlusion pressure and central venous pressure fail to predict ventricular filling volume, cardiac performance, or the response to volume infusion in normal subjects. Crit Care Med 2004;32:691-9.

38 Michard F, Teboul JL. Predicting fluid responsiveness in ICU patients: a critical analysis of the evidence. Chest 2002;121:2000-8.

39 Bednarczyk JM, Fridfinnson JA, Kumar A et al. Incorporating dynamic assessment of fluid responsiveness into goal-directed therapy: a systematic review and meta-analysis. Crit Care Med 2017:45:1538-45. 
40 Pearse RM, Harrison DA, MacDonald N et al. Effect of a perioperative, cardiac output-guided hemodynamic therapy algorithm on outcomes following major gastrointestinal surgery: a randomized clinical trial and systematic review. JAMA 2014;311:2181-90.

41 Elwan MH, Roshdy A, Elsharkawy EM, Eltahan SM, Coats T]. The haemodynamic dilemma in emergency care: Is fluid responsiveness the answer? A systematic review. Scand J Trauma Resusc Emerg Med 2017:25:25.

42 Nunes TS, Ladeira RT, Bafi AT et al. Duration of hemodynamic effects of crystalloids in patients with circulatory shock after initial resuscitation. Ann Intensive Care 2014:4:25.

43 Messina A, Longhini F, Coppo $\mathrm{C}$ et al. Use of the fluid challenge in critically ill adult patients: a systematic review. Anesth Analg 2017:125:1532-43.

44 Biais M, de Courson H, Lanchon R et al. Mini-fluid challenge of $100 \mathrm{ml}$ of crystalloid predicts fluid responsiveness in the operating room. Anesthesiology 2017;127:450-6.

45 Michard F, Boussat S, Chemla D et al. Relation between respiratory changes in arterial pulse pressure and fluid responsiveness in septic patients with acute circulatory failure. Am J Respir Crit Care Med 2000;162:134-8.

46 Lansdorp B, Lemson J, van Putten M] et al. Dynamic indices do not predict volume responsiveness in routine clinical practice. Br J Anaesth 2012;108:395-401.
47 Rutlen DL, Wackers FJ, Zaret BL. Radionuclide assessment of peripheral intravascular capacity: a technique to measure intravascular volume changes in the capacitance circulation in man. Circulation 1981;64:146-52.

48 Wong DH, Tremper KK, Zaccari ] et al. Acute cardiovascular response to passive leg raising. Crit Care Med 1988;16:123-5.

49 Cherpanath TG, Hirsch A, Geerts BF et al. Predicting fluid responsiveness by passive leg raising: a systematic review and meta-analysis of 23 clinical trials. Crit Care Med 2016;44:98191

50 Cherpanath TG, Aarts LP, Groeneveld JA, Geerts BF. Defining fluid responsiveness: a guide to patient-tailored volume titration. J Cardiothorac Vasc Anesth 2014:28:745-54.

51 Wang $\mathrm{CH}$, Hsieh WH, Chou HC et al. Liberal versus restricted fluid resuscitation strategies in trauma patients: a systematic review and meta-analysis of randomized controlled trials and observational studies. Crit Care Med 2014:42:954-61.

Address for correspondence: Dr Elizabeth Sapey, Institute of Inflammation and Ageing, University of Birmingham, Birmingham B15 2GW, UK.

Email: e.sapey@bham.ac.uk

\title{
NGC National

\section{New to systematic reviewing, or need} to improve your critical appraisal skills?

\author{
Our intensive 1-day courses will introduce you to the key \\ principles of critical appraisal and systematic reviewing, \\ and equip you with the basic skills to put your knowledge \\ into practice.
}

> Introduction to critical appraisal > Systematic reviews and meta-analysis in action

Discount available if both courses are booked together.

\section{'Informative and practical' Dr Rajeswari Ramaraj}

\section{NGC Short courses}

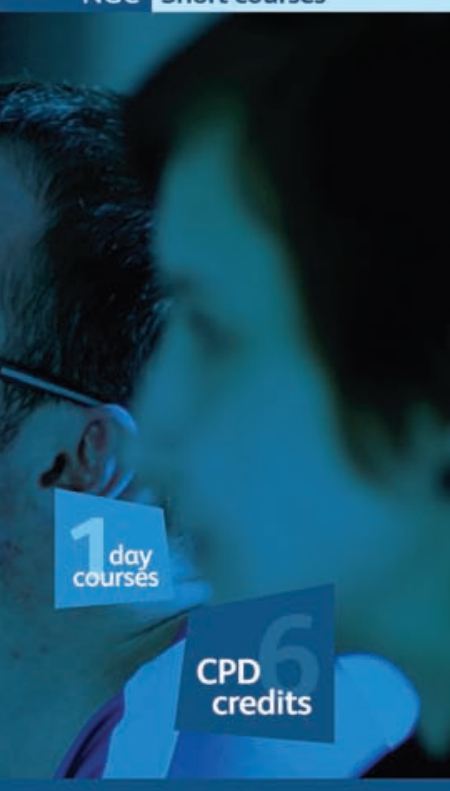

For course dates and more information visit: www.ngc.ac.uk/training-courses 